\title{
INDÚSTRIAS PRODUTORAS DE BIODIESEL: DESTINAÇÃO CORRETA AOS EFLUENTES ATRAVÉS DE IMPLANTAÇÃO DE POLÍTICAS DE PRODUÇÃO MAIS LIMPA (P+L)
}

\section{BIODIESEL PRODUCING INDUSTRIES: CORRECT DESTINATION FOR EFFLUENTS THROUGH THE IMPLEMENTATION OF CLEANER PRODUCTION POLICIES $(P+L)$}

\author{
Carmem Cícera Maria da Silva ${ }^{1}$, Núbia Luiza Corrêa Carvalho², Gustavo Graciano Fonseca³
}

\author{
Submetido em: 26/04/2021 \\ Aprovado em: 16/05/2021
}

e24265

\begin{abstract}
RESUMO
Dentre os biocombustíveis atuais, o biodiesel destaca-se pelo aumento da demanda nos últimos anos e este quadro é consequência de muitas vantagens, principalmente em relação ao meio ambiente. Em todo processo industrial há formação de subprodutos e resíduos que podem se tornar possíveis contaminantes ambientais, não seria diferente na produção de biodiesel. Dentre os resíduos gerados neste processo, os efluentes aparecem em lugar de destaque e essa produção massiva acaba gerando um grande volume. Tais efluentes apresentam em sua composição glicerina, álcool, traços do catalisador entre outros, que impedem seu descarte em rios e solos. Para a realização do tratamento dos efluentes com objetivo de inseri-los dentro das legislações ambientais vigentes, produtores tem encontrado muitos problemas operacionais, econômicos e ambientais. Objetivou-se apresentar um possível meio de atenuação do problema da destinação a ser dada ao efluente gerado pela produção de biodiesel, introduzindo a proposta de políticas de produção mais limpa e aplicando uma solução de reuso aos efluentes. Acrescentando-o à glicerina, que consiste em um subproduto de baixo valor comercial gerado na produção de biodiesel. É necessário destacar que a realização dessa mistura provoca um aumento no volume da glicerina, o que acaba por gerar um aumento no valor financeiro, fato benéfico para as indústrias, uma vez que o efluente representa apenas custos enquanto que a glicerina representa lucro. Os resultados obtidos ao longo da pesquisa mostraram a viabilidade, uma vez que houve um decréscimo considerável dos fatores poluentes envolvidos nas análises.
\end{abstract}

PALAVRAS-CHAVE: Efluentes. Glicerina. Biodiesel

\begin{abstract}
Among the current biofuels, biodiesel stands out for the increase in demand in recent years and this situation is a consequence of many advantages, mainly in relation to the environment. In every industrial process, there is the formation of by-products and residues that can become possible environmental contaminants, it would not be different in the production of biodiesel. Among the waste generated in this process, the effluents appear in a prominent place and this massive production ends up generating a large volume. Such effluents present in their composition glycerin, alcohol, traces of the catalyst among others, which prevent its disposal in rivers and soils. In order to carry out the treatment of effluents with the objective of inserting them within the current environmental legislation, producers have encountered many operational, economic and environmental problems. The objective was to present a possible means of mitigating the problem of the destination to be given to the effluent generated by the production of biodiesel, introducing the proposal of cleaner production policies and

\footnotetext{
1 Pós-Doutoranda em Química na Universidade Federal do Espírito Santo - UFES, Doutora em Ciência e Tecnologia Ambiental (UFGD)

2 Graduada em Engenharia Física na Universidade Estadual do Mato Grosso do Sul

3 Professor na Universidade Federal da Grande Dourados - UFGD, Graduação e Mestrado na área de Engenharia de Alimentos e Doutorado em Biotecnologia, Pós-Doutorado na Universidade de Saarland (Alemanha)
}

RECIMA21 - Ciências Exatas e da Terra, Sociais, da Saúde, Humanas e Engenharia/Tecnologia 


\section{RECIMA21 - REVISTA CIENTÍFICA MULTIDISCIPLINAR ISSN 2675-6218}

INDÚSTRIAS PRODUTORAS DE BIODIESEL: DESTINAÇÃO CORRETA AOS EFLUENTES ATRAVÉS DE IMPLANTAÇÃO DE POLÍTICAS DE PRODUÇÃO MAIS LIMPA (P+L) Carmem Cícera Maria da Silva, Núbia Luiza Corrêa Carvalho, Gustavo Graciano Fonseca

applying a solution of reuse to the effluents. Adding it to glycerin, which consists of a by-product of low commercial value generated in the production of biodiesel. It is necessary to highlight that the realization of this mixture causes an increase in the volume of glycerin, which ends up generating an increase in the financial value, a fact beneficial to the industries, since the effluent represents only costs while the glycerin represents profit. The results obtained throughout the research showed the feasibility, since there was a considerable decrease in the polluting factors involved in the analyzes.

KEYWORDS: Effluents. Glycerin. Biodiesel

\section{Introdução}

O biodiesel é um biocombustível que possui implicações socioeconômicas e contribui efetivamente a reduzir o impacto ambiental associado ao uso de combustíveis fósseis (BARROS et al., 2020). Suas características são semelhantes ao diesel, apresentando segurança ao ser estocado, contendo baixo teor de enxofre, ausência de toxicidade, sendo biodegradável e possuindo menor emissão de poluentes em relação aos combustíveis fósseis. Este biocombustível é formado por ésteres de alquila proveniente da transesterificação de ácidos carboxílicos de cadeia longa, oriundo de origem vegetal ou animal renovável (COSTA, et al., 2020).

Todavia apesar de suas inúmeras vantagens ambientais, sua produção envolve sérios inconvenientes, dentre os quais podemos citar a produção de efluentes, provenientes dos processos de purificação, que envolvem as etapas de lavagem, filtração e secagem do produto final (CORDEIRO et al., 2015). Embora o biodiesel seja considerado uma fonte renovável, estudos apontam que para cada $\mathrm{m}^{3}$ de biodiesel produzido são necessários de 0,2 a 1,2 $\mathrm{m}^{3}$ de água na etapa de lavagem e purificação, o que resulta na produção de grande quantidade de efluente. Neste efluente, geralmente, são encontrados óleos/gorduras, sais, catalisador remanescente, sabão, monoglicerídeos, triglicerídeos, biodiesel e álcool, em concentrações variadas. A presença destes resíduos dificulta o lançamento da água de lavagem diretamente nos corpos hídricos receptores (FERNANDES, et al., 2018).

A etapa de lavagem no processo de produção do biodiesel é uma das mais importantes e críticas, pois tem como objetivo a retirada de resíduos de naturezas distintas, como álcoois, glicerina, catalisador e do próprio óleo usado como matéria-prima. Nesta purificação, o biodiesel utiliza em média um volume três vezes maior de água a cada litro produzido, o efluente resultante do processo se torna quimicamente impróprio a ser despejado em qualquer corpo hídrico (DIAS et al., 2019).

O impacto ambiental causado por tais tipos de efluentes é de avaliação difícil, por causa da variedade de compostos oriundos da matéria-prima, reagentes, produtos e subprodutos do processo. Em geral os efluentes da etapa de purificação de biodiesel apresentam elevada presença de óleos vegetais e outros contaminantes e seu descarte indevido acarretará em um maior custo no tratamento do esgoto dessa região. Um possível descarte in natura causaria impactos nocivos ao meio ambiente (FERREIRA et al., 2019). 


\section{RECIMA21 - REVISTA CIENTÍFICA MULTIDISCIPLINAR ISSN 2675-6218}

INDÚSTRIAS PRODUTORAS DE BIODIESEL: DESTINAÇÃO CORRETA AOS EFLUENTES ATRAVÉS DE IMPLANTAÇÃO DE POLÍTICAS DE PRODUÇÃO MAIS LIMPA (P+L) Carmem Cícera Maria da Silva, Núbia Luiza Corrêa Carvalho, Gustavo Graciano Fonseca

Portanto, estudos apontam como forma possível de atenuação da destinação do efluente através de várias técnicas, tornando-se imprescindível um tratamento deste efluente com possível reaproveitamento nas usinas de biodiesel, ou para seu tratamento posterior por processo biológico em estações de tratamento de esgotos. O tratamento da água de lavagem na produção de biodiesel pode ser feito pela aplicação de processos físicos, biológicos, químicos ou eletroquímicos, tais como a filtração, adsorção, eletrofloculação, coagulação, oxidação avançada, decantação e floculação (DIAS et al., 2019).

Para o procedimento de purificação úmida são acrescidos de solvente pelo menos $4 \%$ do volume total de biodiesel que irá passar pelo processo. A água é o solvente mais utilizado para esse fim, pois tanto a glicerina quanto o álcool são altamente solúveis em água garantindo bons resultados, isso se dá também devido ao baixo custo e a relativa simplicidade do processo. Uma vez misturada a água ao biodiesel, ele passa por um processo de decantação para a total separação de fases e posteriormente de secagem, para que então possa ser dado o seguimento no ciclo de produção e comercialização (BARROS et al., 2020).

As impurezas, por serem na sua maioria polares, ficam todas presentes na água de lavagem, que nesse ponto se tornou um efluente que precisa de tratamento para que possa ser descartado conforme resolução do Conselho Nacional do Meio Ambiente (CONAMA, 2015). Esse tratamento não consiste em algo simples, pois o efluente é constituído por uma grande gama de elementos físicoquimicamente diferentes entre si, o que acaba por gerar a necessidade de um setor específico nas indústrias, demandando de grande quantidade de recursos financeiros por parte das mesmas para que sigam a legislação de descarte de efluentes em águas correntes (DIAS et al., 2019).

A criação de políticas de produção mais limpas tem despertado interesse por parte de nações e governos devido à crescente preocupação com as questões ambientais. Essas políticas compõem uma metodologia que propõe aplicação continuada de uma estratégia ambiental preventiva e integrada aos processos e produtos, a fim de aumentar a eficiência e reduzir os riscos a sociedade e ao meio ambiente, além de minimizar os desperdícios, reduzir custos e alavancar o potencial inovador da organização, visando ganhos de competitividade e a otimização dos processos industriais (BURSZTYN, 2020).

A $P+L$ é uma ferramenta capaz de reduzir impactos identificados nos processos e na atividade, além de proporcionar benefícios financeiros e na imagem da empresa. Sendo que a empresa que deseja trabalhar dentro de um conceito de ecoeficiência, ou seja, que quer produzir "mais e melhor com menos recursos", deve considerar a gestão ambiental como um compromisso para conservar o meio ambiente e limitar as descargas poluentes, além de proporcionar maior segurança no trabalho (NASCIMENTO; SENHORAS, 2019).

Este estudo tem como intuito primordial apresentar um possível meio de atenuação do problema da destinação a ser dada ao efluente gerado pela produção de biodiesel, introduzindo a proposta de políticas de produção mais limpa, de modo que seja feito o reuso do mesmo acarretando em redução de custos ao processo e minimizando possíveis problemas ambientais. 


\section{RECIMA21 - REVISTA CIENTÍFICA MULTIDISCIPLINAR ISSN 2675-6218}

INDÚSTRIAS PRODUTORAS DE BIODIESEL: DESTINAC̄̃O CORRETA AOS EFLUENTES ATRAVÉS DE IMPLANTAÇ̃̃O DE POLÍTICAS DE PRODUÇÃO MAIS LIMPA (P+L) Carmem Cícera Maria da Silva, Núbia Luiza Corrêa Carvalho, Gustavo Graciano Fonseca

\section{Metodologia}

Para a realização desta pesquisa utilizou-se como matéria-prima o óleo de soja degomado, uma amostra de $50 \mathrm{~L}$ foi fornecida por um produtor de biodiesel local. O processo de degomagem consiste em após a extração do óleo, o mesmo é aquecido em água a $60^{\circ} \mathrm{C}$ para que goma seja retirada, sendo a mesma definida como fosfatídeos solúveis em água (REDA, 2007). Optou-se por utilizar o óleo de soja degomado, pois segundo a ANP atualmente no Brasil a maior produção de biodiesel utiliza este tipo de matéria-prima (ANP, 2019).

\section{1 - Transesterificação do Óleo Degomado em escala laboratorial}

Inicialmente o óleo degomado, foi aquecido a $55^{\circ} \mathrm{C}$, temperatura ideal para o favorecimento da reação, e subsequentemente foi acrescentado o metanol e o metilato de sódio, que foi o catalisador escolhido, isento de água, minimizando assim a formação de sabão durante a reação. Essa mesma metodologia foi utilizada nas duas etapas da reação, conforme quantidades estequiométricas apresentadas na Figura 1. Na primeira etapa foi acrescentado metanol, o equivalente a $18 \%$ da massa de óleo e $1 \%$ de metilato de sódio. $\mathrm{Na}$ segunda etapa essas quantidades foram menores, pois grande parcela do óleo já havia reagido e formado biodiesel, necessitando de uma concentração menor de reagente para que a reação se processasse, foi acrescentado então de metanol o equivalente a $7 \%$ da massa inicial de óleo, e 0,5\% de metilato de sódio.

Figura 1: Fluxograma de etapas de transesterificação e decantação

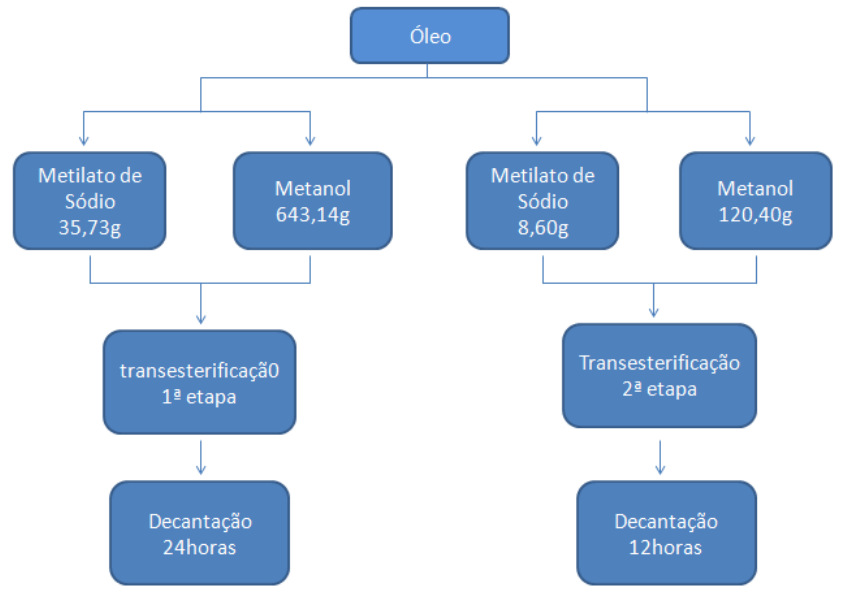

Seguidamente aos reagentes serem adicionados ao óleo, foi controlado o tempo de 60 minutos de agitação, a temperatura constante, para que pudesse ser encaminhado para um período de $24 \mathrm{~h}$ de decantação na primeira etapa e $12 \mathrm{~h}$ na segunda etapa, atingindo uma total separação de fases entre o biodiesel e a glicerina, sendo esta a primeira amostra produzida. Na Figura 2 observase a reação de transesterificação e o processo de decantação em funil de decantação. 


\section{RECIMA21 - REVISTA CIENTÍFICA MULTIDISCIPLINAR ISSN 2675-6218}

INDÚSTRIAS PRODUTORAS DE BIODIESEL: DESTINAC̄̃̃ CORRETA AOS EFLUENTES ATRAVÉS DE IMPLANTACÃO DE POLÍTICAS DE PRODUÇÃO MAIS LIMPA $(\mathrm{P}+\mathrm{L})$ Carmem Cícera Maria da Silva, Núbia Luiza Corrêa Carvalho, Gustavo Graciano Fonseca

Figura 2: Transesterificação e decantação
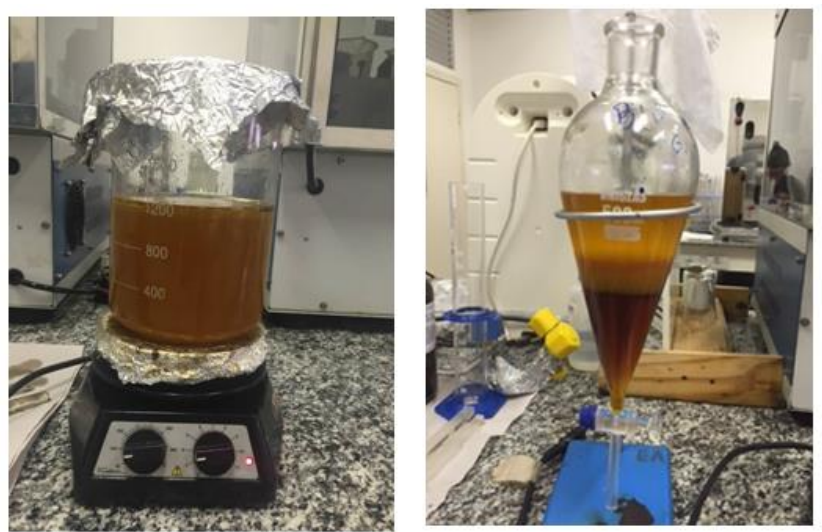

Fonte: Autor

\section{2 - Purificação do biodiesel}

Uma vez finalizada a separação de fases, iniciou-se o procedimento de purificação do biodiesel, que foi constituída pela hidrólise e lavagem do mesmo. Primeiramente, foi acrescentando 400 ppm da massa de biodiesel de ácido fosfórico $\left(\mathrm{H}_{3} \mathrm{PO}_{4}\right)$ para a hidrólise, uma proporção considerada ideal, pois não possui grande influência na acidez, elimina o catalisador e reduz consideravelmente a quantidade de sabão presente. A água utilizada foi retirada de poço artesiano na cidade universitária, no intuito de simular condições aplicadas em processos industriais em larga escala. Para cada lavagem foram acrescentados e misturados $2 \%$ do volume total de biodiesel a ser purificado (Figura 3).

Figura 3: Fluxograma das etapas de purificação e obtenção da mistura (glicerina / efluente 1:1)

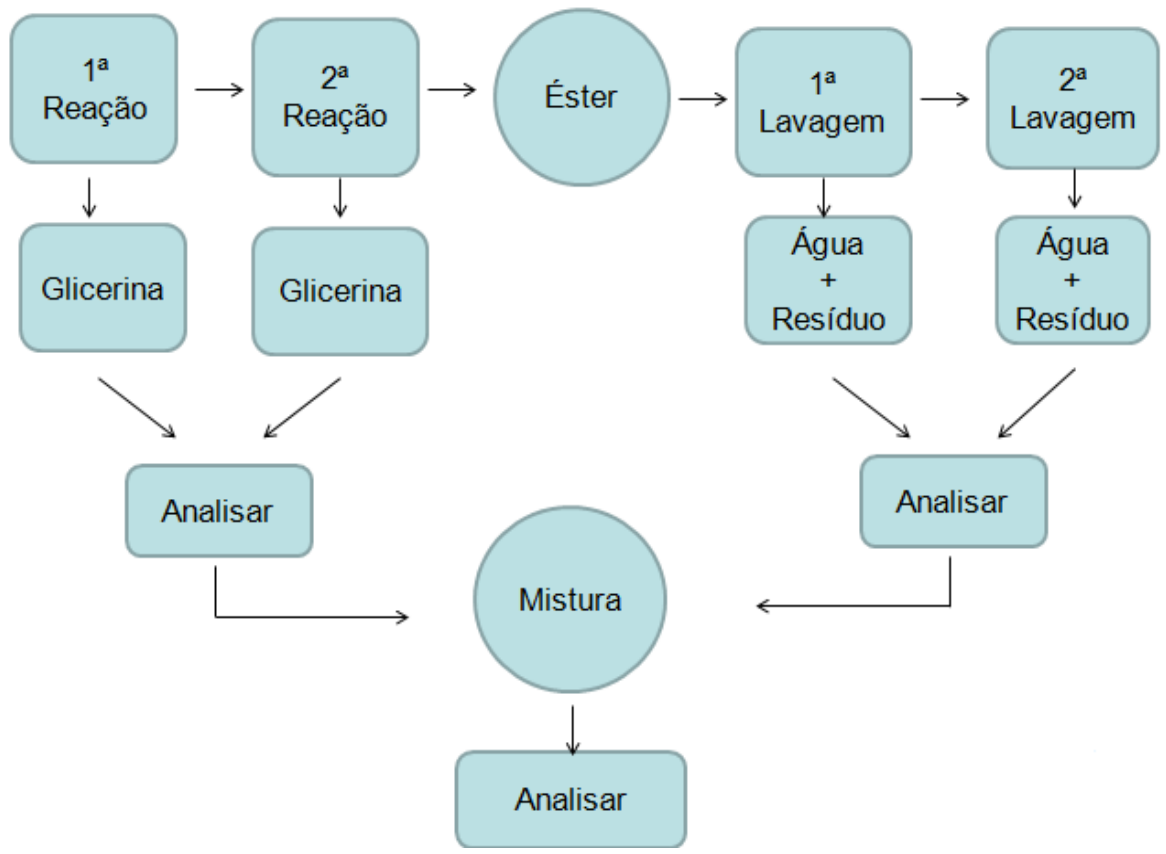




\section{RECIMA21 - REVISTA CIENTÍFICA MULTIDISCIPLINAR ISSN 2675-6218}

INDÚSTRIAS PRODUTORAS DE BIODIESEL: DESTINAC̄̃O CORRETA AOS EFLUENTES ATRAVÉS DE IMPLANTAÇÃO DE POLÍTICAS DE PRODUÇÃO MAIS LIMPA (P+L) Carmem Cícera Maria da Silva, Núbia Luiza Corrêa Carvalho, Gustavo Graciano Fonseca

Após ter sido acrescentada a água em cada lavagem, foi feita uma agitação vigorosa e a mistura foi colocada para decantação por $24 \mathrm{~h}$, para que a separação de fases fosse completa. A Figura 4 apresenta os produtos obtidos na primeira e segunda lavagens.

Figura 4: Detalhamento da etapa de purificação do biodiesel
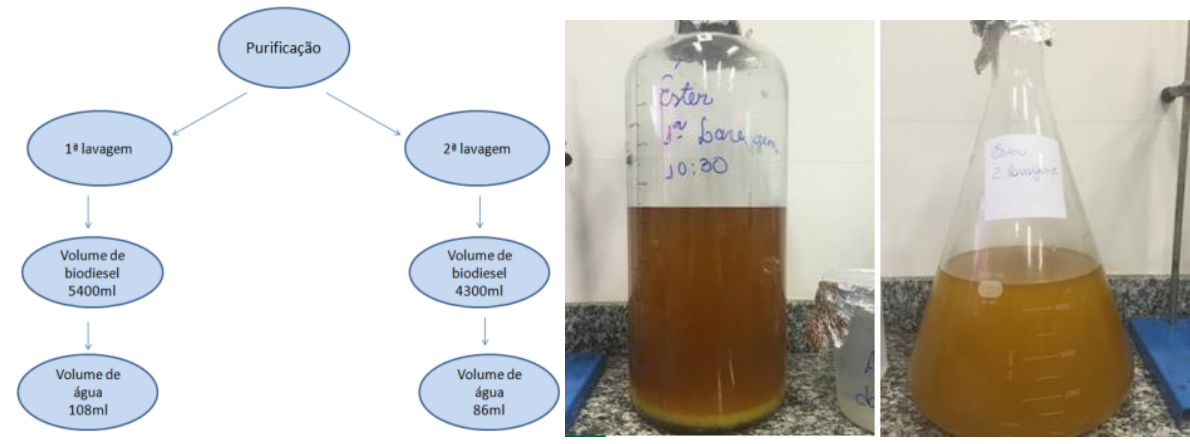

Fonte: Autor

\section{3 - Desestabilização de emulsão}

Após o término da decantação restaram três fases como produto, o biodiesel, a água glicerinosa e uma emulsão, que é definida como a indefinição de fases, ou seja, uma combinação das duas fases previamente citadas, conforme apresentado na Figura 5. Neste caso é necessária a desestabilização das fases, por conter uma considerável quantidade de éster que não poderia ser simplesmente descartada, causando redução de lucros quando analisando o âmbito industrial. Para a eliminação da emulsão, de acordo com Palomino et al., existem diferentes métodos comumente utilizados, sendo o aumento de temperatura, adição de ácido e adição de sais os que mais se destacam (PALOMINO et al.,2012). Sendo o aumento de temperatura e a adição de ácido foram procedimentos adotados nesta etapa (SILVA, 2013).

Figura 5: Emulsão formada entre as fases

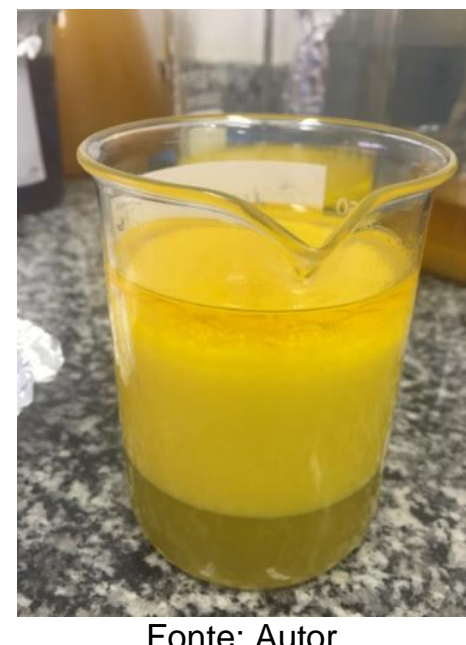

RECIMA21 - Ciências Exatas e da Terra, Sociais, da Saúde, Humanas e Engenharia/Tecnologia 


\section{RECIMA21 - REVISTA CIENTÍFICA MULTIDISCIPLINAR ISSN 2675-6218}

INDÚSTRIAS PRODUTORAS DE BIODIESEL: DESTINAC̄̃̃ CORRETA AOS EFLUENTES ATRAVÉS DE IMPLANTAÇÃO DE POLÍTICAS DE PRODUÇÃO MAIS LIMPA $(\mathrm{P}+\mathrm{L})$ Carmem Cícera Maria da Silva, Núbia Luiza Corrêa Carvalho, Gustavo Graciano Fonseca

\section{4 - Reuso do Efluente}

Uma vez tendo conseguido as amostras de efluente e glicerina, seguiu-se para a metodologia de reuso do primeiro, gerando assim a terceira amostra de interesse da pesquisa. Para isso foram reunidas as amostras previamente obtidas na mesma proporção, formando uma mistura completamente homogênea, em virtude da glicerina ser altamente higroscópica. As três amostras finais obtidas estão apresentadas na Figura 6.

Figura 6: Glicerina, mistura e efluente, respectivamente

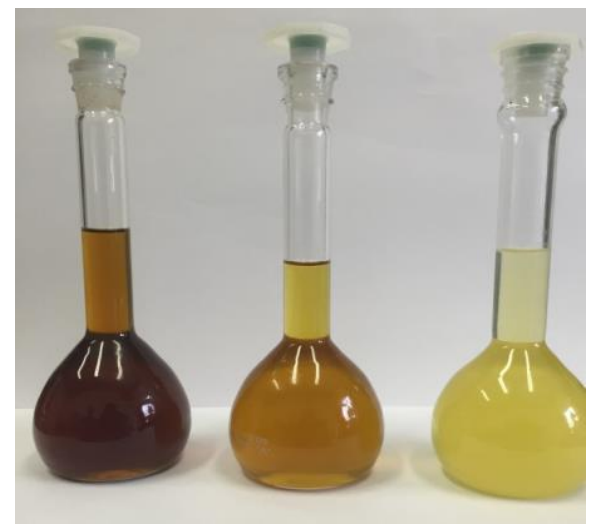

Fonte: Autor

Para o cálculo do rendimento, foi aferida a massa inicial de toda a glicerina obtida no processo e posteriormente, aquecida para evaporação do metanol, pois para o procedimento foram utilizados, do mesmo, o equivalente a $25 \%$ da massa total de óleo para um favorecimento mais efetivo da reação, no entanto, são necessários somente $12 \%$ para a composição estequiométrica dos produtos gerados no processo, causando um excesso de $13 \%$ a ser eliminado. A temperatura utilizada para aquecimento da glicerina foi de $65^{\circ} \mathrm{C}$, dado que este é o ponto de ebulição do metanol. Ao fim de 60 minutos, a massa foi medida novamente, bem como sua densidade determinada.

\section{5 - Caracterização Física e Química}

Para a determinação da viabilidade da proposta, foram analisados aspecto, acidez, alcalinidade livre, alcalinidade combinada, teor de metanol e teor de éster, parâmetros que possibilitam uma melhor compreensão do comportamento dos interferentes ao preparar o reuso do efluente na glicerina, todas foram realizadas em triplicata para que fosse possível fazer o cálculo estatístico posteriormente. A primeira análise realizada foi a de aspecto, avaliando se as amostras estavam límpidas e isentas de impurezas (LII), fator observável pela transparência das mesmas. $\mathrm{O}$ índice de acidez realizou-se segundo a NBR 14448 e a alcalinidade livre e combinada através da titulometria de neutralização. Para a análise do teor de metanol nas amostras, utilizou-se o aquecimento para a evaporação do mesmo. No primeiro momento foram aferidas as massas das amostras de efluente, glicerina e a mistura. Posteriormente foi colocado na estufa a $70^{\circ} \mathrm{C}$ por $1 \mathrm{~h}$ e no 


\section{RECIMA21 - REVISTA CIENTÍFICA MULTIDISCIPLINAR ISSN 2675-6218}

INDÚSTRIAS PRODUTORAS DE BIODIESEL: DESTINAC̄̃̃ CORRETA AOS EFLUENTES ATRAVÉS DE IMPLANTAÇÃO DE POLÍTICAS DE PRODUÇÃO MAIS LIMPA (P+L) Carmem Cícera Maria da Silva, Núbia Luiza Corrêa Carvalho, Gustavo Graciano Fonseca

dessecador por 30 minutos, logo após este procedimento, as amostras foram aferidas novamente para que fosse possível verificar a massa do metanol evaporado. O mesmo processo foi repetido até massa constante, indicando que possivelmente todo o metanol já havia sido evaporado. O teor de éster presente nas amostras foi analisado ao colocar as mesmas na centrífuga por 10 minutos, para separação de fases através da diferença de densidade, caso ainda houvesse resquícios de éster alquílico nas amostras.

\section{6 - Pesquisas de Custos}

Foi realizada uma pesquisa para levantamento de custos em diversos produtores listados em todo o Brasil pela Agência Nacional de Petróleo e Biocombustíveis (ANP), no intuito de determinar os custos operacionais com produtos químicos e energia consumida, no tratamento de efluentes oriundos da produção de biodiesel.

\section{Resultados e Discussão}

3.1 - Desestabilização de emulsão, rendimento, caracterização física e química e pesquisas de custos da política $\mathrm{P}+\mathrm{L}$

A emulsão foi submetida a uma temperatura de $90^{\circ} \mathrm{C}$ por 30 minutos, conforme Figura 7 , resultando numa separação de fases inferior a desejada. Sabendo que sua massa era de aproximadamente $110 \mathrm{~g}$, foi acrescentado ácido fosfórico aos poucos, de modo que se observou que o equivalente a $0,80 \%$ da massa da emulsão foi a quantia suficiente para que a desestabilização total ocorresse, possibilitando a separação das fases e obtenção do efluente a ser analisado posteriormente.

Figura 7: Desestabilização da emulsão obtida

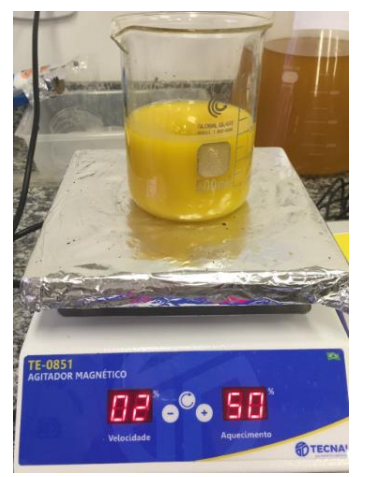

Fonte: Autor

Os valores obtidos nos cálculos do rendimento estão apresentados na Tabela 1. Com os valores obtidos pelo cálculo do rendimento, foi possível determinar que o percentual de glicerina gera na transesterificação corresponde a 11,36\%, valor de boa expressão, tendo em vista sua considerável aproximação com o percentual de 10\% determinado pela estequiometria (MENDES, D. B.; SERRA, 2012). Essa diferença deve-se ao fato de que as impurezas ainda estão presentes no glicerol, que 


\section{RECIMA21 - REVISTA CIENTÍFICA MULTIDISCIPLINAR ISSN 2675-6218}

INDÚSTRIAS PRODUTORAS DE BIODIESEL: DESTINAÇÃO CORRETA AOS EFLUENTES ATRAVÉS DE IMPLANTACÃO DE POLÍTICAS DE PRODUÇÃO MAIS LIMPA $(\mathrm{P}+\mathrm{L})$ Carmem Cícera Maria da Silva, Núbia Luiza Corrêa Carvalho, Gustavo Graciano Fonseca

não passou por um procedimento de purificação, e que apresentou um teor de 4,86\% de metanol em sua composição anterior a evaporação. Da massa inicial de óleo utilizada, 4,58\% é referente ao efluente, o que equivale a $40 \%$ da massa final da glicerina.

Tabela 1: Rendimento reacional, teor de glicerol e metanol da glicerina.

\begin{tabular}{ccccc}
$\begin{array}{c}\text { Massa } \\
\text { inicial (g) }\end{array}$ & $\begin{array}{c}\text { Massa } \\
\text { final (g) }\end{array}$ & $\begin{array}{c}\text { Teor de glicerina } \\
\text { inicial (\%) }\end{array}$ & $\begin{array}{c}\text { Teor de glicerina } \\
\text { final (\%) }\end{array}$ & $\begin{array}{c}\text { Teor de } \\
\text { metanol (\%) }\end{array}$ \\
\hline 579,53 & 405,73 & 16,22 & 11,36 & 4,86 \\
\hline
\end{tabular}

$\mathrm{Na}$ análise de aspecto notou-se que as amostras não estavam límpidas e isentas de impurezas. A amostra da mistura entre o efluente e a glicerina apresentou cristais de sal, devido à hidrólise ocorrida ao acrescentar ácido fosfórico para eliminar o sabão, conforme previamente citado. Esta reação gera sal e água como produtos, sendo que o primeiro ficou em suspensão. A presença de impurezas demonstra a necessidade de que em um segundo momento as amostras deverão passar por um processo de purificação, para que aumente seu valor agregado, bem como sua aplicabilidade. Os dados quantitativos obtidos nas análises estão apresentados na Tabela 2.

Tabela 2: Parâmetros físico-químicos da glicerina, efluente e mistura

\begin{tabular}{cccc}
\hline Parâmetros & Glicerina & Efluente & $\begin{array}{c}\text { Mistura (glicerina e } \\
\text { efluente 1:1) }\end{array}$ \\
\hline $\begin{array}{c}\text { Acidez }(\%) \\
\text { Alcalinidade livre } \\
(\text { meq } / \mathrm{g})\end{array}$ & $0,400 \pm 0,010^{\mathrm{A}}$ & $55,00 \pm 0,02^{\mathrm{A}}$ & $28,00 \pm 0,00^{\mathrm{B}}$ \\
$\begin{array}{c}\text { Alcalinidade combinada } \\
(\%)\end{array}$ & $11,60 \pm 0,30^{\mathrm{A}}$ & $6,60 \pm 0,01^{\mathrm{C}}$ & $0,019 \pm 0,001^{\mathrm{B}}$ \\
Teor de metanol $(\%)$ & $17,62 \pm 2,21^{\mathrm{C}}$ & $75,82 \pm 0,23^{\mathrm{A}}$ & $7,80 \pm 0,01^{\mathrm{B}}$ \\
\hline
\end{tabular}

Para a análise de acidez, a quantidade presente na glicerina foi inferior ao valor detectável através do método utilizado. Em se tratando do efluente, efetuar a mistura do mesmo com a glicerina foi benéfico, uma vez que diminuiu seu teor de acidez em $56 \%$. Quanto a alcalinidade livre que representa o catalisador ativo, não houve detecção do mesmo presente no efluente pelo método de análise utilizado, porém houve uma diminuição de 0,381 meq/g na quantia presente na glicerina após realizar a mistura. A respeito da quantidade de sabão existente nas amostras, o teste de alcalinidade combinada mostrou que houve uma queda de $54 \%$ no teor presente na glicerina ao efetuar a mistura com o efluente, de modo a ser necessária uma menor quantidade de produtos para a posterior eliminação desse sabão. E o resultado obtido em relação ao teor de metanol também foi consideravelmente positivo, pois houve uma queda de aproximadamente 30 pontos percentuais na 


\section{RECIMA21 - REVISTA CIENTÍFICA MULTIDISCIPLINAR ISSN 2675-6218}

INDÚSTRIAS PRODUTORAS DE BIODIESEL: DESTINAC̄̃O CORRETA AOS EFLUENTES ATRAVÉS DE IMPLANTAÇÃO DE POLÍTICAS DE PRODUÇÃO MAIS LIMPA $(P+L)$ Carmem Cícera Maria da Silva, Núbia Luiza Corrêa Carvalho, Gustavo Graciano Fonseca

quantidade apresentada pelo efluente após ser misturada, valendo destacar que para nenhuma das análises realizadas, houve correlação de resultados obtidos pela análise estatística.

Da análise de teor de éster, obteve-se que nenhuma das amostras analisadas acusou a presença de ésteres, o que caracteriza um resultado positivo por demonstrar que houve uma total separação de fases no processo de decantação (PALOMINO-ROMERO et al, 2012) não tendo sido perdido quantidade considerável de biodiesel, conforme Figura 8.

Figura 8: Teor de ésteres nas amostras de efluentes, glicerina e mistura, respectivamente
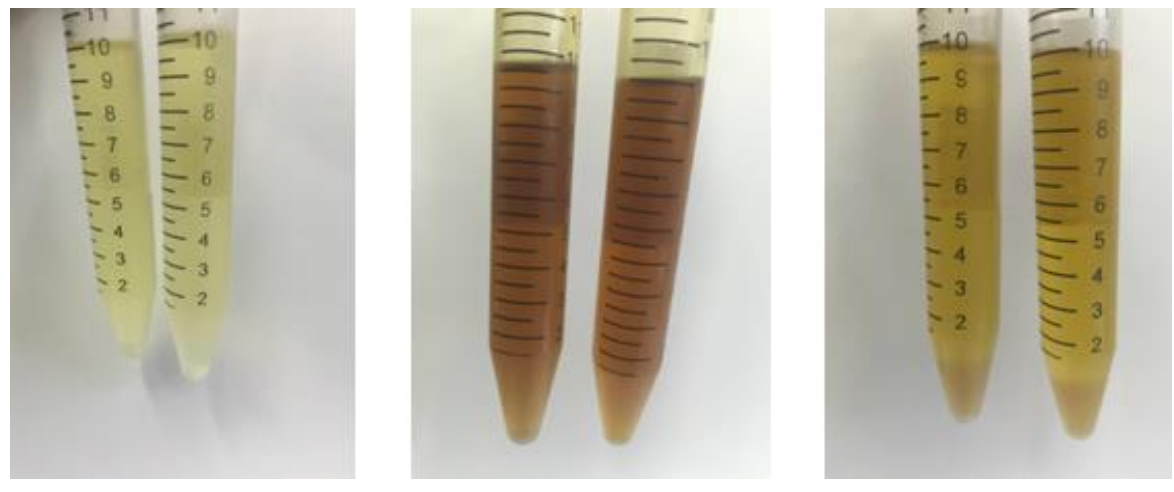

Fonte: Autor

Sobre a pesquisa de custos realizada com as cinco indústrias de biodiesel a respeito dos custos com os respectivos tratamentos de efluentes, somente uma indústria local retornou com a planilha de custos dos produtos utilizados para o tratamento do efluente gerado na produção, os mesmos estão apresentados na Tabela 3.

Tabela 3: Custos com produtos da ETE de uma empresa de biodiesel.

\begin{tabular}{lcccc}
\hline \multicolumn{1}{c}{ Produtos } & $\begin{array}{c}\text { Valor } \\
(\mathbf{R} \$)\end{array}$ & Dosagem $/ \mathbf{m}^{\mathbf{3}}$ & $\begin{array}{c}\text { Concentração } \\
(\%)\end{array}$ & $\begin{array}{c}\text { Custo } \\
\left(\mathbf{R} \mathbf{\$} / \mathbf{m}^{\mathbf{3}}\right)\end{array}$ \\
\hline Coagulante & 2,65 & 1,5 & 100 & 3,98 \\
Polímero & 23,73 & 10 & 0,12 & 0,28 \\
Ácido fosfórico & 9,60 & 0,3 & 85 & 2,45 \\
\cline { 3 - 5 } & & & Total & 6,71 \\
\hline
\end{tabular}

Esta indústria produz, quando operando normalmente, $45 \mathrm{~m}^{3}$ de biodiesel por dia. Foram realizados cálculos quanto a quantidade de efluente gerada diariamente e foi possível constatar que ela produz uma média de $2,061 \mathrm{~m}^{3}$. Essa quantidade é relativamente pequena, comparada a outras empresas de maior porte e capacidade de produção. Contudo, os produtos utilizados para o tratamento deste efluente acarretam em um custo mensal de aproximadamente $R \$ 415,00$ para a mesma.

Essa quantidade produzida diariamente gera por volta de $5,11 \mathrm{~m}^{3}$ de glicerina, que detém um baixo valor comercial, girando em torno de $\mathrm{R} \$ 1.000,00$ a tonelada, de acordo com pesquisas de 


\section{RECIMA21 - REVISTA CIENTÍFICA MULTIDISCIPLINAR ISSN 2675-6218}

INDÚSTRIAS PRODUTORAS DE BIODIESEL: DESTINAC̄̃̃ CORRETA AOS EFLUENTES ATRAVÉS DE IMPLANTAÇÃO DE POLÍTICAS DE PRODUÇÃO MAIS LIMPA $(\mathrm{P}+\mathrm{L})$ Carmem Cícera Maria da Silva, Núbia Luiza Corrêa Carvalho, Gustavo Graciano Fonseca

mercado no estado de Mato Grosso do Sul. A realização da mistura aumentaria em média 40\% o volume da mesma, acarretando num maior lucro devido ao aumento da quantidade a ser comercializada. Levando em conta o fato de ser uma empresa de pequeno porte e que não foi considerado para efeito de cálculos os gastos relacionados aos recursos humanos e aos equipamentos utilizados durante o processo, pode-se considerar as despesas com o tratamento do efluente relativamente altas, e que são custos fixos mensais, tendendo somente a aumentar, conforme aumente sua capacidade de produção.

Ao analisar a produção de empresas de grande porte como as três A, B e C, citadas na Figura 9, cujos nomes forma omitidos por questão de sigilo e que são apresentadas nos dados da ANP como ativas (ANP, 2016).

Figura 9: Dados da produção de biodiesel de três empresas.

\begin{tabular}{|c|c|}
\hline $\begin{array}{l}\text { EMPRESA A } \\
\text { AUTORIZAÇÃO: № } 370 \text { de 12/8/2011, DOU de 15/8/2011 } \\
\text { OBJETO DA AUTORIZAÇÃO: Comercialização ESPECIFICAÇÃO: Comercialização } \\
\text { SITUAÇÃO: Ativa }\end{array}$ & CAPACIDADE DE PRODUÇÃO (m3/dia): 1352 \\
\hline $\begin{array}{l}\text { EMPRESA B } \\
\text { AUTORIZAÇÃO: № } 50 \text { de 02/2/2012, DOU de 10/2/2012 } \\
\text { OBJETO DA AUTORIZAÇÃO: Operação } \\
\text { SITUAÇÃO: Ativa }\end{array}$ & CAPACIDADE DE PRODUÇÃO (m3/dia): 1033 \\
\hline $\begin{array}{l}\text { EMPRESA C } \\
\text { AUTORIZAÇÃO: № } 570 \text { de 20/12/2011, DOU de 22/12/2011 } \\
\text { OBJETO DA AUTORIZAÇÃO: Operação } \\
\text { SITUAÇÃO: Ativa }\end{array}$ & CAPACIDADE DE PRODUÇÃO (m3/dia): 900 \\
\hline
\end{tabular}

Fonte: ANP (2016)

Foram estimados o volume de efluente gerado e os custos relacionados ao seu tratamento, podendo observar que resultam num valor muito mais expressivo. Para via de cálculos, foi seguida a mesma linha de custo disponibilizada pela empresa local, conforme previamente mencionado. Os resultados obtidos estão apresentados na Tabela 4.

Tabela 4: Valores estimados da geração de efluente e seu tratamento das empresas A, B e C.

\begin{tabular}{cccc}
\hline Empresa & $\begin{array}{c}\text { Capacidade de Produção } \\
\text { diária }\left(\mathbf{m}^{\mathbf{3}}\right)\end{array}$ & $\begin{array}{c}\text { Efluente gerado } \\
\text { diariamente }\left(\mathbf{m}^{3}\right)\end{array}$ & $\begin{array}{c}\text { Custo mensal de } \\
\text { tratamento }(\mathbf{R} \$)\end{array}$ \\
\hline A & 1352 & 65 & $13.084,50$ \\
B & 1033 & 50,20 & $10.105,26$ \\
C & 900 & 43,74 & $8.804,86$ \\
\hline
\end{tabular}




\section{RECIMA21 - REVISTA CIENTÍFICA MULTIDISCIPLINAR ISSN 2675-6218}

INDÚSTRIAS PRODUTORAS DE BIODIESEL: DESTINAC̃̃O CORRETA AOS EFLUENTES ATRAVÉS DE IMPLANTAÇÃO DE POLÍTICAS DE PRODUÇÃO MAIS LIMPA (P+L)

Com esses dados nota-se que uma empresa que produz uma quantidade massiva de biodiesel diariamente, acaba produzindo também uma grande quantidade de efluente de forma que seu custo mensal se torna alto. O que possibilita uma maior clareza ao analisar a relevância do estudo aqui apresentado, que além de ter um foco na gestão ambiental, causa um impacto positivo também nos aspectos financeiros das indústrias.

A pesquisa foi feita em escala laboratorial, porém buscando uma similaridade com a metodologia usualmente utilizada em uma indústria de biodiesel, resultando aproximadamente numa quantidade de efluente proporcional ao volume produzido em escala industrial. A água residual gerada do procedimento de purificação do biodiesel contém diversos interferentes que acarretam num alto grau de poluição caso sejam descartados no meio ambiente. É necessário também levar em consideração o custo acarretado pelo tratamento desse efluente, devido ao fato da obrigatoriedade em seguir a legislação para que a empresa seja autorizada a efetuar a produção, causando um aumento na quantidade de materiais, equipamentos e pessoal necessários para o funcionamento de um setor dedicado a esse fim, a estação de tratamento de efluentes, fato este que desperta a necessidade de uma alternativa de destinação para os efluentes, buscando uma maior praticidade e diminuição dos gastos.

Os resultados obtidos ao longo da pesquisa mostraram a viabilidade desse procedimento, uma vez que houve um decréscimo considerável dos fatores poluentes envolvidos nas análises, tornando possível concluir que existe a possibilidade de uma redução na necessidade de realizar o tratamento do efluente, podendo fazer uma mistura dele com o subproduto da transesterificação, consumindo completamente seu volume ao utilizar uma proporção de 1:1, pois sua quantia gerada é menor que a de glicerina, sendo que esta acaba tendo seu volume amplificado.

As pesquisas de custos apontaram que a empresa analisada, possui um gasto de pelo menos $\mathrm{R} \$ 415,00$ mensais com o tratamento de seu efluente, sendo que a mesma produz aproximadamente $1350 \mathrm{~m}^{3}$ de biodiesel mensalmente, o que caracteriza uma capacidade de produção pequena, quando comparada a outras empresas presentes no ramo de biocombustíveis, como as citadas neste trabalho por empresas A, B e C, em que a quantidade produzida, bem como a de efluente gerada e recursos necessários para o tratamento, muito maior. Esses custos poderiam ser reduzidos ao efetuar o que este trabalho propõe, pois, a mistura causaria uma redução nos índices poluentes e aumentaria o volume de glicerina. É necessário destacar que posteriormente a mistura passe por um procedimento de purificação, que se dá, porém, de forma mais simples e com menos custos que o tratamento do efluente, para que então essa glicerina possa ser comercializada no mercado.

\section{Considerações Finais}

A realização deste estudo deu-se em escala laboratorial, porém buscando uma similaridade com a metodologia usualmente utilizada em uma indústria de biodiesel, resultando naturalmente numa quantidade de efluente proporcional ao volume produzido. A água residual gerada (efluente) do procedimento de purificação do biodiesel contém diversos interferentes que ocasionariam um alto 


\section{RECIMA21 - REVISTA CIENTÍFICA MULTIDISCIPLINAR ISSN 2675-6218}

INDÚSTRIAS PRODUTORAS DE BIODIESEL: DESTINAÇÃO CORRETA AOS EFLUENTES ATRAVÉS DE IMPLANTAÇÃO DE POLÍTICAS DE PRODUÇÃO MAIS LIMPA (P+L) Carmem Cícera Maria da Silva, Núbia Luiza Corrêa Carvalho, Gustavo Graciano Fonseca

grau de poluição caso fossem descartados no meio ambiente. É necessário também levar em consideração o custo acarretado pelo tratamento desse efluente, devido ao fato da obrigatoriedade em seguir a legislação para que a empresa seja autorizada a efetuar a produção. Causando um aumento na quantidade de materiais, equipamentos e pessoal necessários para o funcionamento de um setor dedicado a esse fim, a estação de tratamento de efluentes, fatos que despertam a necessidade de uma alternativa de destinação para o mesmo, buscando uma maior praticidade e diminuição dos gastos.

Esta pesquisa teve por objetivo apresentar uma proposta de redução dos impactos causados pelo descarte dos efluentes, bem como dos custos para o tratamento deste, fazendo seu reuso na glicerina, também causando um aumento de seu valor comercial que usualmente é pouco valorizada no mercado. Os resultados obtidos ao longo da pesquisa mostraram a viabilidade desse procedimento, uma vez que houve um decréscimo considerável dos fatores poluentes envolvidos nas análises, tornando possível concluir que existe a possibilidade de uma redução na necessidade de realizar o tratamento do efluente, podendo fazer uma mistura dele com o subproduto da transesterificação, consumindo completamente seu volume ao utilizar uma proporção de 1:1, pois sua quantia gerada é menor que a de glicerina, sendo que esta acaba tendo seu volume aumentado.

As pesquisas de custos apontaram que a empresa analisada, possui um gasto de pelo menos $\mathrm{R} \$ 415,00$ mensais com o tratamento de seu efluente, sendo que a mesma produz aproximadamente $1350 \mathrm{~m}^{3}$ de biodiesel mensalmente, o que caracteriza uma capacidade de produção pequena, quando comparada a outras empresas presentes no ramo de biocombustíveis. Esses custos poderiam ser reduzidos ao efetuar o que este trabalho propõe, pois, a mistura causaria uma redução nos índices poluentes e aumentaria o volume de glicerina. É necessário destacar que posteriormente a mistura passe por um procedimento de purificação, que se dá, porém, de forma mais simples e com menos custos que o tratamento do efluente, para que então essa glicerina possa ser comercializada no mercado.

Como limitação a essa proposta de reuso do efluente na glicerina, tem-se a questão de estrutura industrial de armazenamento dessa mistura (tancagem) todos os produtores de biodiesel tiveram suas plantas industriais projetadas para armazenar somente glicerina, como haverá aumento de volume, os mesmos deveriam investir em tanques de armazenamento para essa mistura, mas seria somente um único investimento inicial e posteriormente se pagaria rapidamente com a redução dos custos de efluentes e aumento da glicerina comercializada.

\section{AGRADECIMENTOS}

Universidade Federal da Grande Dourados - UFGD, Universidade Estadual do Mato Grosso do Sul UEMS, FUNDECT, CAPES e CNPq 


\section{RECIMA21 - REVISTA CIENTÍFICA MULTIDISCIPLINAR ISSN 2675-6218}

INDÚSTRIAS PRODUTORAS DE BIODIESEL: DESTINAC̄̃O CORRETA AOS EFLUENTES ATRAVÉS DE IMPLANTACÃO DE POLÍTICAS DE PRODUÇÃO MAIS LIMPA $(\mathrm{P}+\mathrm{L})$ Carmem Cícera Maria da Silva, Núbia Luiza Corrêa Carvalho, Gustavo Graciano Fonseca

\section{REFERÊNCIAS}

ANP. Agência Nacional do Petróleo, Gás Natural e Biocombustíveis. Disponível em: http://www.anp.gov.br/biocombustíveis. Acesso em: dez. 2019.

ANP. Agência Nacional do Petróleo, Gás Natural e Biocombustíveis. Disponível em: http://www.anp.gov.br/biocombustíveis. Acesso em: mar. 2016.

BARROS, L. H. C.; PURIFICAÇÃO, M. C.; CAMPANHA, N.; SILVA, T. F. H.; SANTOS, A. G. Biodiesel do óleo da semente de pinha produzido por reação via aquecimento e ultrassom. South American Journal of Basic Education, Technical and Technological, v. 7, n. 1, p. 94-107, 2020.

COSTA, Lucas G. et al. Quantificação do teor de biodiesel de Crambe em misturas com diesel utilizando espectroscopia mir e seleção de variáveis. Quím. Nova, São Paulo, v. 43, n. 6, p. 723-728, jun. 2020.

BURSZTYN, MARCEL. Energia solar e desenvolvimento sustentável no Semiárido: o desafio da integração de políticas públicas. Estud. av., São Paulo, v. 34, n. 98, p. 167-186, abr. 2020.

CONSELHO NACIONAL DO MEIO AMBIENTE-CONAMA. Resolução n. 430. Brasília, 2011.

CORDEIRO, R. B.; ALEXANDRE, J. I. S.; SILVA, J. P. F.; SALES, D. C. S.; CAVALCANTI, L. A. P. Purificação e reutilização de águas residuárias da produção de biodiesel por meio da eletrofloculação. Rev. Bras. Gest. Amb. Sustent., v. 2, n. 2, p. 51-58, 2015.

DIAS, Guilherme et al. Biorremediação de efluentes por meio da aplicação de microalgas - uma revisão. Quím. Nova, São Paulo, v. 42, n. 8, p. 891-899, aug. 2019.

FERNANDES, Carlos H. M. et al. Tratamento eletroquímico de efluente da produção de biodiesel usando um eletrodo do tipo ade: Ti/lrO2-Nb2O5. Quím. Nova, São Paulo, v. 41, n. 1, p. 17-22, Jan. 2018.

FERREIRA, T. F., RIBEIRO, C. B., GUERI M. V. D., BURATTO W. G., PAVANELLO, G. P., AYOUB, J. P., BESINELLA G. B., STROPARO, C. E., Estudo comparativo dos processos de adsorção e fotooxidação no tratamento do efluente de biodiesel, R. gest. sust. ambient., Florianópolis, v. 8, n. 1, p.472-482, jan./mar. 2019.

MENDES, D. B.; SERRA, J. C. V. Glicerina: uma abordagem sobre a produção e o tratamento. Revista Liberato, v. 13, n. 20, p.1-9, 2012.

NASCIMENTO, F. L.; SENHORAS, E. M. Produção mais limpa, logística reversa e consórcios públicos intermunicipais na gestão de resíduos sólidos em Roraima. BOLETIM DE CONJUNTURA, Boa Vista, ano I, v. 1, n. 1, 2019.

NBR 14448 - Produtos de petróleo - Determinação do índice de acidez pelo método de titulação potenciométrica. 2005.

PALOMINO-ROMERO, Joel A. et al. Tratamentos dos efluentes gerados na produção de biodiesel. Química Nova, Aracaju, v. 35, n. 2, p. 367-378, set. 2012.

REDA, S. Y.; CARNEIRO, P. I. B. Óleos e gorduras: Aplicações e implicações. Analytica, v. 17, n. 60, 2007.

SILVA, E. M. S.; SILVA, A. C. Desestabilização de emulsões de óleo em água pela adição da "baba" do quiabo (Abelmoschus esculentus) extraída a quente. Goiania: CONPEEX, UFG, 2013. 